\title{
Mechanical characterization of porous nano-thin films by use of atomic force acoustic microscopy
}

\author{
M. Kopycinska-Müller ${ }^{1,2}$, A. Clausner ${ }^{1}$, K.-B. Yeap ${ }^{1}$, B. Köhler ${ }^{1}$, N. Kuzeyeva ${ }^{1}$, S. Mahajan ${ }^{3}$, \\ T. Savage ${ }^{3}$, E. Zschech ${ }^{1,2}$, K.-J. Wolter ${ }^{2}$ \\ ${ }^{1}$ Fraunhofer Institute for Ceramic Technologies and Systems - Material Diagnostics IKTS-MD, Maria Reiche \\ Str. 2, 01109, Dresden, Germany \\ ${ }^{2}$ Faculty of Electrical Engineering and Information Technology, Technical University Dresden, Helmholtz Str. \\ 18, 01069, Dresden, Germany \\ ${ }^{3}$ SBA Materials, Inc., 9430-H San Mateo Blvd, NE Albuquerque, NM 87113, USA
}

\begin{abstract}
The indentation modulus of thin films of porous organosilicate glass with a nominal porosity content of $30 \%$ and thicknesses of $350 \mathrm{~nm}, 200 \mathrm{~nm}$, and $46 \mathrm{~nm}$ is determined with help of atomic force acoustic microscopy (AFAM). This scanning probe microscopy based technique provides the highest possible depth resolution. The values of the indentation modulus obtained for the $350 \mathrm{~nm}$ and $200 \mathrm{~nm}$ thin films were respectively $6.3 \mathrm{GPa} \pm 0.2 \mathrm{GPa}$ and 7.2 $\mathrm{GPa} \pm 0.2 \mathrm{GPa}$ and free of the substrate influence. The sample with the thickness of $46 \mathrm{~nm}$ was tested in four independent measurement sets. Cantilevers with two different tip radii of about $150 \mathrm{~nm}$ and less than $50 \mathrm{~nm}$ were applied in different force ranges to obtain a result for the indentation modulus that was free of the substrate influence. A detailed data analysis yielded value of $8.3 \mathrm{GPa} \pm 0.4 \mathrm{GPa}$ for the thinnest film.

The values of the indentation modulus obtained for the thin films of porous organosilicate glasses increased with the decreasing film thickness. The stiffening observed for the porous films could be explained by evolution of the pore topology as a function of the film thickness. To ensure that our results were free of the substrate influence, we analyzed the ratio of the sample deformation as well as the tip radius to the film thickness. The results obtained for the substrate parameter were compared for all the measurement series and showed, which ones could be declared as free of the substrate influence.
\end{abstract}

Keywords: AFM, AFAM, thin films, porous materials, elastic properties 


\section{Introduction}

The integration of new materials into novel microelectronic products is always challenging. Particularly, porous organosilicate glass used as insulating dielectrics in on-chip interconnect stacks must meet a complex set of materials properties including dielectric permittivity and Young's modulus. The incorporation of $\mathrm{CH}_{2}$ - and $\mathrm{CH}_{3}$ - groups and pores into silica networks significantly reduces the values for the Young's modulus and hardness of the thin film material [1]. However, the mechanical properties of the so-called ultra-low $\mathrm{k}$ (ULK) materials can be improved by adjusting the pore concentration, as well as their shape, size, and arrangement, referred to as pore topology. The stability of the pore topology across the film thickness becomes yet another factor that needs to be characterized as the miniaturization tendencies progress in the nano-electronics industry.

Brillouin light scattering, surface acoustic wave spectroscopy [2], picosecond ultrasonic methods [3], and ellipsometric porosimetry [4] methods have been used to determine the values of the Young's modulus of very thin films. The values obtained are free of the substrate influence and represent the mean value integrated from a relatively large area. One can use contact methods such as nanoindentation [5] and methods based on atomic force microscopy (AFM) [6] to determine mechanical properties from a confined region. The exact diameter of the contact area is defined by the contact mechanics models and may vary from few tens of $\mathrm{nm}$ to few hundreds of $\mathrm{nm}$ depending on the elastic properties of the indenter and the sample, indenter's geometry and the load applied to maintain the contact between the bodies.

State of the art nanoindentation instruments are capable of performing indentations with depth of $20 \mathrm{~nm}$ and less. To determine the indentation modulus of a thin film without considering the influence of the substrate, the indentation depth must be significantly less than the film thickness. Furthermore, as soon as the film thickness becomes less than $1 \mu \mathrm{m}$, additional factors have to be taken into account, for example the relative difference in the elastic properties of the film and the substrate as well as the ratios of the contact radius and indentation depth to the film thickness $[5,7]$.

Small indentation depth experiments are not trivial [8] and in case of very thin films they do not always guarantee a result independent on the substrate properties. Thus, numerous models accounting for substrate influence on the load-displacement (P-h) curves measured on thinfilm samples in nanoindentation experiments have been developed during the previous 30 years. Doerner and Nix [9] proposed a method for the determination of the Young's modulus from the initial part of a standard load-displacement curve measured in nanoindentation. Their empirical model took into account the ratio between the film thickness and the effective indentation depth. Their expression also included constants that had to be determined empirically from P-h curves measured on a thin film sample as well as on a bulk substrate. The model proposed by King [10] took into account the influence of the tip geometry on the measured P-h curve. His results showed that the effective elastic properties of the filmsubstrate system depended on the contact radius to film thickness ratio $a / t$. Moreover, he determined the values of the geometry factor for flat punches of different geometries (circular, triangular and square) as a function of the $a / t$ ratio. A closed form solution for the contact 
compliance of a film-substrate system was proposed by Gao [11]. The so-called perturbation analysis is based on the known elastic solutions for a reference homogenous body made either of the film or the substrate material. The particular elastic properties of the reference homogenous material determine the limits of the perturbation solution. However, Gao's approach is valid only for film-substrate system with a maximum relative difference in their elastic properties of about $200 \%-300 \%$. In the cases, where the substrate is much stiffer than the film, Swain and Mencik [12] adapted a model developed for large-scale indentation of rubber sheets published by Waters [13].

The models proposed by $[9,10,11,13,14]$ can be expressed in a general form:

$\frac{1}{M_{e f f}}=\frac{1}{M_{s}}[1-f(t, a, h, \alpha)]+\frac{1}{M_{f}} f(t, a, h, \alpha)$,

The effective indentation modulus $M_{\text {eff }}$ is the modulus of the film-substrate system determined at given load conditions, $M_{s}$ is the indentation modulus of the substrate, and $M_{f}$ is the indentation modulus of the film. The function $f(t, a, h, \alpha)$ describes how the substrate properties affect the effective indentation modulus of the system as a function of the film thickness $t$, contact radius $a$ or indentation depth $h$ and a parameter $\alpha$ that is related to the tip geometry.

The determination of the mechanical properties of thin films from the unload part of the P-h curve obtained in the plastic-elastic regime is limited due to possible film cracking and delamination, reverse plasticity, creep, or viscoplasticity. A method developed and tested by Schwarzer and Chudoba [15, 16, 17] allows characterization of very thin films (in nm range) from the analysis of elastic interaction between a relatively large spherical indenter (from few to several $\mu \mathrm{m}$ in radius) and a film-substrate system. Their method is based on an analytical stress-strain solution of a contact problem for a layered half space system under defined load conditions and calculating the resulting surface displacement. Those are then compared to the displacement measured in the experiment. The main advantage of this method is that it allows for application of relatively large loads that can be maintained with high precision by a nanoindenter. However, the stability of the measurement in the elastic regime is paid by a loss of the lateral resolution.

Another approach was proposed by Li and Vlassak in [18]. They implemented Yu's elastic solution into an analysis procedure that allowed for the estimation of the contact area from the P-h curve obtained on a thin film sample in a range of the indentation depth that could be easily maintained by the instrument. Li's method allows for large mismatch in the mechanical properties of the film and the substrate. Bull [19] proposed a very simplified approach to evaluate the modulus of a film-substrate system where no empirical geometry factors are needed. However, this model has also problems for systems with large differences in the Young's moduli of the film and the substrate.

Methods based on atomic force microscopy (AFM) [6] offer the ability to control the static loads in the range of single $\mathrm{nN}$ and exert localized pressures with very sharp tips with radii below $20 \mathrm{~nm}$ yielding unparalleled spatial resolution for contact probing. AFM-based methods can be used to obtain color-coded maps of such quantities as tip-sample adhesion, 
Young's modulus, storage and loss moduli, and indentation modulus [20, 21, 22, 23, 24, 25, 26, 27]. There are several methodologies utilizing AFM instruments for mechanical characterization of materials. The most direct way to study mechanical properties of a sample is to record the force displacement curves and interpret them in terms of contact mechanics [28]. The force-displacement curve can be measured during the standard approach of the cantilever to the sample surface at each point of the image (force volume method) [28], or by monitoring the cantilever deflection as the z-offset of the piezotube is modulated at frequencies below the first natural frequency of the cantilever (Pulsed-force mode) [29]. In addition, the analysis of the cantilever vibrations at its flexural and torsional modes is used to recalculate the calibrated force-displacement curves in the so-called torsional harmonic cantilever mode [20].

The AFM methods based on force-distance measurements are most effective when applied to compliant materials such as biological tissue or polymer. To access the information on the mechanical properties of materials such as glasses, metals and ceramics one must restore to dynamic modes of AFM operation. The term "dynamic" relates to vibrations of the AFM cantilever at frequencies greater than that of the first free flexural mode. Dynamic modes encompass several methods such ultrasonic force microscopy (UFM) [30], ultrasonic atomic force microscopy (UAFM) [31], and atomic force acoustic microscopy (AFAM) [32] that was further developed into contact-resonance force microscopy (CR-FM, and CR-AFM) imaging methods [33, 34].

There are several publications reporting successful applications of the AFAM methods in the field of thin film characterization. For example, the indentation modulus of niobium films with thicknesses below $300 \mathrm{~nm}$ was determined in single point measurements [35], as well as from stiffness maps [36] obtained by use of AFAM and CR-FM methods, respectively. Stan and Cook [34] successfully characterized $100 \mathrm{~nm}$ thin films of granular gold with CR-AFM. Indentation moduli determined for $800 \mathrm{~nm}, 200 \mathrm{~nm}$, and $50 \mathrm{~nm}$ thin films of nanocrystalline nickel showed the influence of the increasing intercrystalline volume on the effective elastic properties of the sample [37]. In these studies, the investigated films were thick enough to allow their direct characterization and to neglect the influence of the substrate.

AFAM and similar methods were also used to characterize mechanical properties of films thinner than $50 \mathrm{~nm}$. Hurley et al. used the CR-FM method to study the adhesion at an interface of a $20 \mathrm{~nm}$ thin film of gold and silicon substrate [38]. Muraoka applied cantilevers modified with point weights to characterize $10 \mathrm{~nm}$ thin diamond-like-carbon layers by use of the AFAM method [39]. The AFAM method was also used to determine the indentation modulus of silicon oxide films with thicknesses ranging from $8 \mathrm{~nm}$ to $28 \mathrm{~nm}$ [40]. In the studies presented in $[39,40]$ the substrate influence was considered. The results presented in this paper were obtained by combining the ability of the AFAM method to characterize very thin film [40] with that to determine the indentation modulus of low and ultra-low k materials as reported in $[41,42,43]$.

\section{Samples}


The porous organosilicate glass films tested in this study were prepared in a self-assembly process [44] from an alcohol based hydrolyzed sol of silica modified with $\mathrm{CH}_{2} / \mathrm{CH}_{3}$ groups and containing aligned amphiphilic triblock copolymers. The solution containing the same concentration of the copolymer was spin-coated on single crystal silicon coated previously with a nano-thin layer of SiC:N yielding the layers investigated in this study. The copolymer was evaporated during thermal curing leaving behind an organized pore structure. The pore size was expected to be between 1.5 and $3 \mathrm{~nm}$. It is an assumed value, as it was determined by use of ellipsometric porosimetry for samples prepared by the same method but with thickness of about $600 \mathrm{~nm}$ [42]. The nominal porosity content of the $600 \mathrm{~nm}$ sample was 30\%. The samples investigated in this study were prepared from identical solutions, thus it was assumed that the porosity content was also $30 \%$ regardless of the films thickness. The parameters describing the thin film samples were given by the manufacturer. The tested samples were very smooth. The roughness confidents determined from tapping mode images with scan size $500 \mathrm{~nm} \times 250 \mathrm{~nm}$ were $0.2 \mathrm{~nm}, 0.2 \mathrm{~nm}$, and $0.3 \mathrm{~nm}$ for ULK-350, ULK-200 and ULK-46 sample, respectively. Table I presents the names and the thicknesses of the tested samples. A chemical vapor deposited OSG (CVD OSG) sample with an indentation modulus $\mathrm{M}_{\text {ref }}=7$ GPa determined by nanoindentation [8] was used as reference sample for the AFAM measurement procedure [42].

Table I. The thicknesses of the tested organosilicate films.

\begin{tabular}{|l|l|l|l|}
\hline Name & ULK-350 & ULK-200 & ULK-46 \\
\hline Film thickness & $350 \mathrm{~nm}$ & $200 \mathrm{~nm}$ & $46 \mathrm{~nm}$ \\
\hline
\end{tabular}

\section{Method}

The AFAM methods is based on the determination of resonance frequencies of an AFM cantilever vibrating in contact with the sample surface for at least two modes. The values of the contact resonance frequencies are compared to those of the corresponding free modes. The model describing the dynamic behavior of the AFM cantilever treats it as micro-sized rectangular beam that is rigidly fixed on one end and spring-coupled on the other end. For systems, where elastic forces dominate tip-sample interaction forces, the stiffness of the model spring can be interpreted as the tip-sample contact stiffness $k^{*}$. For our calculations we have used an analytical model described in detail [45]. This model describes the influence of the normal stiffness and position of the tip on the value of the resonance frequencies of the system. In our experiments we treated the tip position parameter as unknown. Thus, contact resonance frequencies for two modes were measured and used to determine both the tip position parameter and the corresponding contact stiffness. The lateral contact stiffness, as well as normal and lateral damping were neglected. Information on further models describing the AFM cantilever in contact can be found in [46].

Models for contact mechanics define the relationship between the contact stiffness and the elastic properties of the sample. In most general terms, for contact geometries of three or fourfold symmetries, $k^{*}$ depends on the diameter of the contact area and the reduced Young's modulus $E^{*}$. 
The reduced Young's modulus $E^{*}$ is calculated from the indentation moduli of the tip $\left(\mathrm{M}_{\mathrm{tip}}\right)$ and the sample $\left(\mathrm{M}_{\mathrm{sam}}\right)$ [47]:

$\frac{1}{E^{*}}=\frac{1}{M_{\text {tip }}}+\frac{1}{M_{\text {sam }}}$

In case of bulk, homogenous and elastically isotropic materials $M_{\text {sam }}=\frac{1-v_{\text {sam }}}{E_{\text {sam }}}$, where $v_{\text {sam }}$ and $E_{\text {sam }}$ are the Poisson's ratio and Young's modulus of the sample [48]. In case of more complex systems such as nano-thin films or samples with buried defects, where the tip-sample interaction volume is not homogeneous it is better to use term of effective indentation modulus $M_{\text {eff }}=M_{\text {sam }}$.

Figure 1 presents the schematic representation of an AFAM system configured for singlepoint spectroscopy measurements. The contact resonance frequencies are excited by an ultrasonic transducer placed below the sample. The longitudinal waves actuate vertical movement of the sample surface triggering the bending vibrations of the cantilever. The amplitude of the vibrations depends strongly on the excitation frequency and is measured by a laser beam deflected from the cantilever to a photodiode detector. In our system, the deflection component of the photodiode signal is analyzed by use of fast Fourier transform (FFT) methods [27, 49].

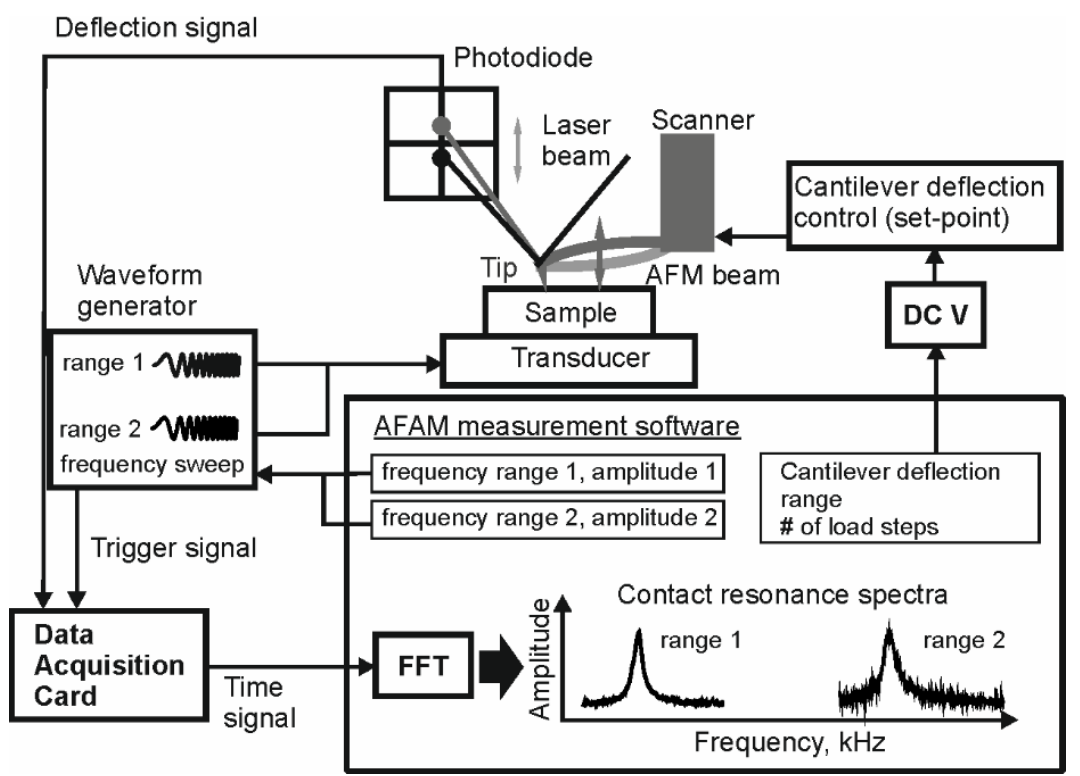

Fig. 1. Schematic representation of an AFAM system capable of excitation and detection of resonance frequencies for at least two modes simultaneously. The contact resonance spectra are recorded as a function of the cantilever static deflection.

The results presented in this study were obtained using AFAM in the single-point spectroscopy mode. In this mode, the resonance frequencies are measured for at least two modes in air (free resonance frequencies) and then in contact with the sample surface as a function of the applied static load. In our system, the range of the static load applied during 
the measurement is defined by the user by setting the lowest and highest deflection values, as well as the number of the measurement steps. Then, the so-called "set-point" voltage value that controls the cantilever deflection is applied externally from a DC voltage generator to the AFM signal access box. A single change of the static load is followed after $100 \mathrm{~ms}-200 \mathrm{~ms}$ waiting time by the measurement of the contact resonance frequencies. The measurements can be performed as a function of the increasing (load) as well as decreasing load (unload). The details concerning the simultaneous excitation of the two or more contact resonance spectra can be found in [49]. Once the contact resonance frequencies are measured, the values of the tip-sample contact stiffness $k^{*}$ can be calculated. General AFAM methodology for calculation of the indentation modulus of the sample is described in [50].

\section{Measurement and data evaluation procedure}

We have used the AFAM method previously to determine the values of the indentation modulus for porous organosilicate glasses. The values of the indentation modulus obtained with the AFAM method were in a very good agreement with those obtained by nanoindentation [42]. Thus, we used the same experimental procedure to characterize the mechanical properties of the porous ultra-thin films. In the first experiment series a cantilever with spring constant $k_{c}$ of about $27 \mathrm{~N} / \mathrm{m}$ and a rounded tip sensor was applied to all ULK samples studied in this paper. Preliminary analysis of the data revealed that the range of the applied loads, while suitable to characterize the films with thicknesses of $350 \mathrm{~nm}$ and $200 \mathrm{~nm}$, was too large for the ULK-46nm sample. Thus, the load range was reduced. Our goal was to obtain a data set that was free of the substrate influence for all three samples. To achieve that for the thinnest film, we had to apply tips with smaller radii as well as cantilevers with spring constant value below $10 \mathrm{~N} / \mathrm{m}$. In total, four cantilevers of different tip radii and different values of $k_{c}$ were used to characterize the properties of the ULK-46nm sample.

The values of the free resonance frequencies were used together with the geometrical dimensions of the cantilevers to calculate the corresponding values of their spring constant by use of the Sader method [51]. Table II contains the information on the ranges of the estimated values of the static loads used during the AFAM measurements. As it can be seen, the load applied differed depending on the cantilever type and the sample thickness. For a standard AFAM experiment performed on a bulk sample, we tend to choose a maximum range of the cantilever deflection that would yield elastic stiffness curves for load and unload as to ensure a stable and reproducible measurement. In case of thin-film systems, one must compromise the stability of the measurement for sake of the reduction in the influence of the substrate elastic properties on the measurement result.

Next to the range of the applied load, the tip radius is yet another important parameter determining the size of the tip-sample interaction volume. We used two types of tips, namely rounded tips with radii in the range of $100 \mathrm{~nm}$ and standard "sharp" tips with radii below 50 $\mathrm{nm}$. To analyze the influence of the substrate on the results obtained in this study, we needed more detailed information on the tip geometry than only nominal values of their radii. As it was shown in [52, 53 ], AFM tips are rarely spherical and after being used in contact mode their geometry resemble a flat punch with rounded edges. Thus to obtain the most accurate results for the indentation modulus by use of AFAM and similar methods, one uses a 
reference sample with elastic properties as similar to those of the unknown sample as possible, especially when assuming Hertz-contact conditions. Then the "effective" tip radius $R_{e f f}$ is the same on both samples. The reference sample used in this study had the indentation modulus of $7 \mathrm{GPa}$ and thus we could use the values of the reference contact stiffness $k_{r e f}^{*}$ to calculate the effective tip radius interacting with the thin ULK films. Similar evaluation of the contact geometry was done by Stan in [43]. In the cited study, the values of the contact stiffness measured on polystyrene reference sample were used to calculate the contact radius and the results obtained were in good agreement with those evaluated from an SEM image of the blunt tip used in the measurements.

Due to non-ideal tip geometry, calculation of the tip radius with help of standard macroscopic models for contact mechanics is not trivial. For example, using the individual values of the contact stiffness determined for a reference sample to calculate the tip radius from equation $R=\frac{k_{r e f}^{* 3}}{6 F E_{r e f}^{*}}$ will yield results that depend both on the applied load and the modulus of the reference sample used [54]. Thus, we applied the approach used by nanoindentation [8] and calculated the effective tip radius from the dependence between the load and the sample deformation $F(\delta)$. Parameter $\delta_{0}$ is a fitting parameter that accounts for the presence of the tip-sample adhesion forces or deviation of the surface from the assumed perfect half space.

$F=4 / 3 \sqrt{R_{\text {eff }}} E_{\text {ref }}^{*}\left(\delta-\delta_{0}\right)^{3 / 2}$

The deformation of the sample was calculated from the values of the tip-sample contact stiffness and the applied static loads.

$\delta=1.5 \frac{F}{k_{r e f}^{*}}$

The results of the fitting procedure performed for each of the measurement series are presented in Table II.

Table II. List of the AFM cantilevers used in this study. The parameters characteristic for each cantilever were the values of the free resonance frequencies and the spring constants. The measurements were performed in different ranges of the static load to minimalize the influence of the substrate on the results obtained for $M_{\text {eff }}$ on the ULK-46 sample. The values of the effective tip radius were determined from corresponding sets of the reference measurements.

\begin{tabular}{|c|c|c|c|c|c|}
\hline$\#$ & $\begin{array}{c}\text { Effective tip } \\
\text { radius } R_{\text {eff }}, \mathrm{nm}\end{array}$ & $\begin{array}{c}\text { Free resonance } \\
\text { frequencies, } \mathrm{kHz}\end{array}$ & $\mathrm{k}_{\mathrm{c}}, \mathrm{N} / \mathrm{m}$ & $\begin{array}{c}\text { Static load range } \\
F, \mathrm{nN}\end{array}$ & Samples tested \\
\hline \multirow{3}{*}{1} & $315 \pm 8$ & \multirow{2}{*}{$\mathrm{f}_{1}{ }^{0}=156.7$} & & (a) $650-4750$ & ULK-350 \\
\cline { 2 - 4 } & $305 \pm 7$ & $\mathrm{f}_{2}{ }^{0}=970.0$ & 27 & (b) $650-4750$ & ULK -200 \\
\cline { 2 - 4 } & $406 \pm 13$ & & (c) $270-3350$ & ULK -46 \\
\hline \multirow{2}{*}{2} & $52 \pm 1$ & $\begin{array}{c}\mathrm{f}_{1}{ }^{0}=167.6 \\
\mathrm{f}_{2}{ }^{0}=1030.7\end{array}$ & 32 & $200-1400$ & ULK -46 \\
\hline 3 & $241 \pm 2$ & $\mathrm{f}_{1}{ }^{0}=82.0$ & 4.3 & $280-645$ & ULK -46 \\
\hline
\end{tabular}




\begin{tabular}{|l|l|c|l|l|l|}
\hline & & $\mathrm{f}_{2}{ }^{0}=542.2$ & & & \\
$\mathrm{f}_{3}{ }^{0}=1519.2$ & & & \\
\hline & & $\mathrm{f}_{1}{ }^{0}=59.8$ & & & \\
$\mathrm{f}_{2}{ }^{0}=382.4$ & 1.9 & $65-190$ & ULK -46 \\
$\mathrm{f}_{3}{ }^{0}=1082.7$ & & & \\
\hline
\end{tabular}

The single point measurements follow always a well-defined sequence. The measurement series start with a reference sample. Then the measurements on the unknown sample alternate with additional reference measurements. Thus, we can monitor the tip wear and account for it by evaluating the effective indentation modulus of the sample with help of the prior and ensuing reference measurement. In addition, we monitored very carefully the pull-off forces for each measurement. They differed depending on the sample. We have used the model proposed by Derjaguin-Muller-Toporov (DMT) [55] to account for the differences in the total forces acting for the tip. For a tip with spherical tip geometry the reduced Young's modulus of the sample $E_{\text {sam }}^{*}$ is calculated from:

$E_{\text {sam }}^{*}=E_{\text {ref }}^{*}\left(\frac{k_{\text {sam }}^{*}}{k_{\text {ref }}^{*}}\right)^{1.5}\left(\frac{F_{r e f}}{F_{\text {sam }}}\right)^{0.5}$

$F_{r e f}$ and $F_{\text {sam }}$ are the total forces acting between the tip and the reference and unknown sample, respectively.

\section{Experimental results and data analysis}

Figure 2 presents values of the effective indentation modulus $M_{\text {eff }}$ calculated for the ULK $350 \mathrm{~nm}$ and ULK - 200nm samples. The cantilever used in these measurements had a spring constant of about $27 \mathrm{~N} / \mathrm{m}$ and a rounded sensor tip. The curves presented in the figure represent the mean values calculated as a function of the applied static load from 20 single point measurements. The error bars are the corresponding standard deviations. The values of the effective indentation modulus determined for the ULK -350nm sample are lower than those determined for the ULK -200nm sample. For both samples, the values of $M_{\text {eff }}$ determined during load tend to be lower than those determined during unload. The difference between the load and unload values increases with decreasing static load and is less than the error bar values. In addition, the values of $M_{\text {eff }}$ increase with increasing static load, but only for the lower values of the static loads. The $M_{\text {eff }}$ values determined at static loads greater than $2000 \mathrm{nN}$ remain constant. In most of the cases, the load-unload curves determined in AFAM measurements overlap, indicating elastic interaction. Factors responsible for the unload values of the contact stiffness being greater than the load ones can be various, such as a small tip wear, small plastic deformation of the sample, or in case of porous material collapsing of pores. In addition, we have observed that for the same sample the load values of the contact stiffness can be either smaller or greater than the unload values depending on the load rate and size of the tip used in the experiment. 


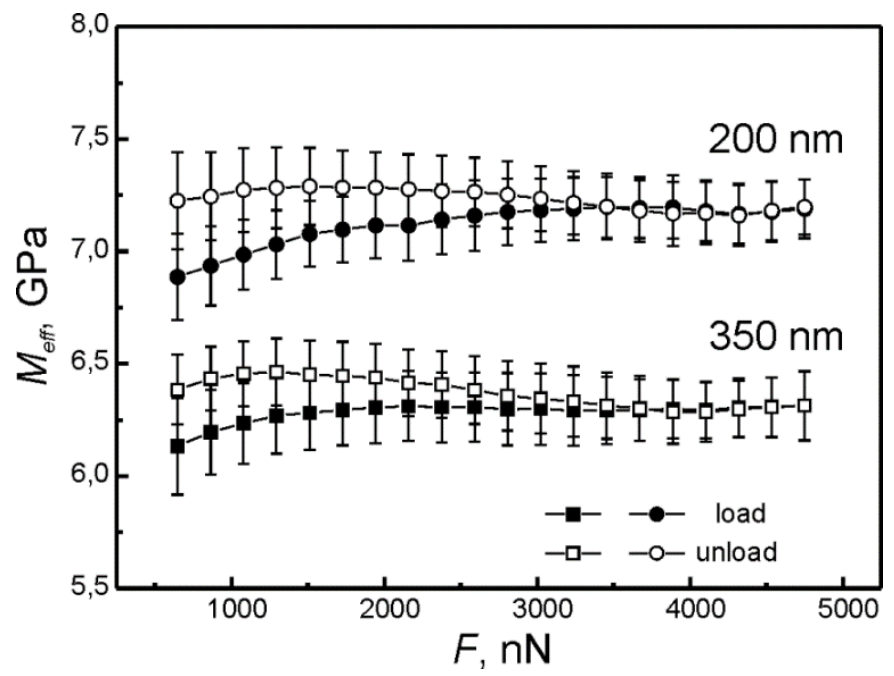

Fig. 2. Values of the effective indentation modulus $M_{\text {eff }}$ determined for the ULK -350 $\mathrm{nm}$ and ULK -200 nm samples as a function of increasing and decreasing load. A cantilever with a rounded tip-sensor was used.

As it can be seen in fig. 2, the values of the effective indentation modulus determined for the ULK -350nm and ULK -200nm films differ significantly, despite the same nominal porosity. The lack of a pronounced dependence of $M_{e f f}$ on the applied static load indicates that the results are not influenced by the elastic properties of the substrate. The initial increase in the values of the effective indentation modulus and presence of the hysteresis in the load-unload curve is caused most probably by a small plastic deformation of the film's upper layer.

Similar behavior was observed for porous organosilicate glasses with a porosity of $30 \%$ and a thickness of about $700 \mathrm{~nm}$ [42]. The mean values of $M_{\text {eff }}$ calculated for the $350 \mathrm{~nm}$ and 200 $\mathrm{nm}$ thin films from all the measurement points acquired during the measurements were 6.3 $\mathrm{GPa} \pm 0.2 \mathrm{GPa}$ and $7.2 \mathrm{GPa} \pm 0.2 \mathrm{GPa}$, respectively.

The values of the effective indentation modulus determined for the ULK -46nm sample are presented in figs. 3 and 4 . In the first attempt, we used the rounded tip and relatively large values of the static load ranging from $270 \mathrm{nN}$ to $3350 \mathrm{nN}$. The results obtained in this series of measurements are presented in fig. 3a. The load-unload curve represents the mean values of $M_{\text {eff }}$ calculated as a function of the static load from 20 single point measurements. The error bars represent the corresponding values of the standard deviation. In contrast to those presented in fig. 2, the curve presented in fig. 3a shows a very strong dependence on the applied static load. The mean values of $M_{\text {eff }}$ increase from $14 \mathrm{GPa}$ to $18 \mathrm{GPa}$ during load and decrease to about $15 \mathrm{GPa}$ during unload. The difference between the load and unload values of $M_{\text {eff }}$ has similar character to that visible in fig. 2, namely it is smaller than the values of the standard deviation of the corresponding mean value and it decreases with the increasing value of the static load.

The strong increase in the values of the effective indentation modulus suggests a substrate influence. To minimize it, we had to reduce the size of the stress field expanding into the sample. This can be done by applying an AFM tip with a smaller tip radius and/or lower values of the static load. To ensure that radical change in the tip geometry will not influence 
the results obtained for the indentation modulus of the samples, we performed an additional test. The test sample was also a film of porous organosilicate glass but with a nominal porosity of $40 \%$ and thickness of $350 \mathrm{~nm}$. Due to the higher porosity content, the modulus of the test sample was lower than that of the ULK-350 sample. Thus, the test sample would deform more under the same load and the influence of the tip geometry would be even more pronounced. The measurement and data evaluation procedures were the same as for the thinfilm samples. The mean value of the indentation modulus determined for the test sample with the blunt tip (cantilever \#1) in the force range from $650 \mathrm{nN}$ to $4750 \mathrm{nN}$ was $3.6 \mathrm{GPa} \pm 0.2$ $\mathrm{GPa}$. Then we used a cantilever equipped with a standard sensor tip having radius in the range from $30 \mathrm{~nm}$ to $50 \mathrm{~nm}$ (cantilever\#2). The force ranged from $160 \mathrm{nN}$ to $1500 \mathrm{nN}$. The mean value of the indentation modulus obtained with the sharp tip was $3.9 \pm 0.1 \mathrm{GPa}$. The result obtained for the indentation modulus with the sharp tip was in a good agreement with that obtained with the blunt tip. The small difference of $0.3 \mathrm{GPa}$ could be caused by differences in the actual and the assumed tip geometries, and the corresponding tip-sample contact conditions. We will use this difference in further analysis of the experimental data obtained for ULK-thin film samples sample. Namely, any difference in the data obtained with blunt and sharp tips smaller than $0.3 \mathrm{GPa}$ will be treated as negligible.

We repeated the measurements on sample ULK-46nm, but this time we used the cantilever \#2 with a smaller tip radius. The applied static load ranged from $200 \mathrm{nN}$ to $1400 \mathrm{nN}$. We performed 24 single point measurements. The curve representing the mean values of the $M_{\text {eff }}$ as a function of the applied static load is presented in fig. 3b. The error bars represent the values of the corresponding standard deviation. As it can be seen in fig. $3 \mathrm{~b}$, the mean values of $M_{\text {eff }}$ obtained for the ULK $-46 \mathrm{~nm}$ sample with standard tip still show dependence on the applied static load. The values of $M_{\text {eff }}$ increase from 8.6 GPa to $10.2 \mathrm{GPa}$ during load and decrease to 9.2 during unload. The maximal value of $M_{\text {eff }}$ determined during this series of measurements is less than the minimal value of $M_{\text {eff }}$ determined for the same sample with help of the rounded tip.
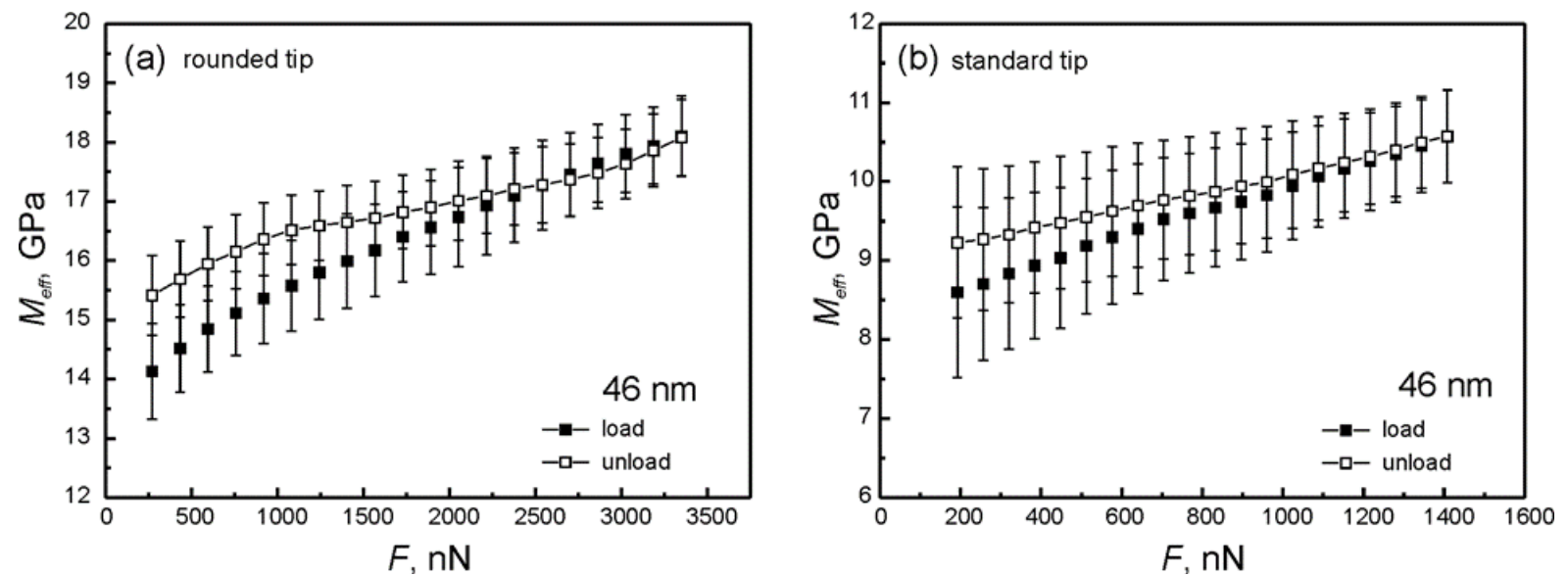

Fig. 3. Values of the effective indentation modulus $M_{\text {eff }}$ determined for the ULK $-46 \mathrm{~nm}$ sample as a function of increasing and decreasing load with (a) rounded and (b) standard sensor tip. 
The influence of the substrate on the effective elastic properties of thin-film system can be accounted for, as it was shown in $[9,10,11]$. As the AFAM method is similar in many aspects to nanoindentation method, we used the previously developed models and adapted them to our needs [40]. The effective indentation modulus $M_{\text {eff }}$ of a system consisting of a thin film with the thickness $t$ and indentation modulus $M_{f}$ and a substrate with the indentation modulus $M_{s}$ examined with a tip of geometry described by parameter $\alpha$ can be calculated from:

$\frac{1}{M_{e f f}}=\frac{1}{M_{S}}+\left(\frac{1}{M_{f}}-\frac{1}{M_{S}}\right) e^{-\alpha \frac{F^{2 / 3}}{t^{2}}}$

The details concerning the model and its accuracy go beyond the scope of this paper and will be discussed separately. We fitted eq. (7) to our experimental data with help of Origin Lab software. We treated the indentation modulus of the substrate and the film thickness as known and the indentation modulus of the film and the geometry parameter $\alpha$ were obtained from the best fit parameters. The fit was performed on curves calculated from averaged load and unload parts of the curves presented in figs. $3 \mathrm{a}$ and $3 \mathrm{~b}$. The fit procedure yielded different values of the indentation modulus of the film, namely of $13.8 \mathrm{GPa} \pm 0.1 \mathrm{GPa}$ and $8.3 \mathrm{GPa} \pm$ $0.1 \mathrm{GPa}$ for the curves obtained with the rounded and standard tip, respectively. Both values are greater than those obtained for the ULK -350nm and ULK -200nm samples. However, the discrepancy between the values obtained with the tips of different curvature, indicates that our empirical model does not describe at least one of the curves correctly. Thus, we repeated the measurements at even lower values of the static loads, but with sensor tips similar to those used previously, namely with a large rounded tip as well as with a standard sharp tip.

We performed additional two series of single point AFAM measurements. Each series consisted of six measurements on the ULK $-46 \mathrm{~nm}$ sample alternated with reference measurements on the OSG sample. The individual values of the effective indentation modulus $M_{\text {eff }}$ were averaged as a function of the applied static load and presented with the corresponding values of the standard deviation in fig. 4. Figure 4 a presents the load-unload curve obtained on the ULK $-46 \mathrm{~nm}$ sample with the rounded tip. The applied static load ranged from $280 \mathrm{nN}$ to $645 \mathrm{nN}$. The maximal value of the applied static load was about 5 times smaller than that applied in previous measurement series. Nevertheless, the values of $M_{e f f}$ obtained within this measurement series showed dependence on the applied static load and were very similar to these presented in fig. 3a despite the significant reduction of the applied static load. The fit of eq. 7 to the experimental data presented in fig. 4a yielded value of 14.5 $\mathrm{GPa} \pm 0.2 \mathrm{GPa}$ for the indentation modulus of the film.

We reduced the applied static load even further when performing the measurements with the standard tip. This time the applied load ranged from $65 \mathrm{nN}$ to $190 \mathrm{nN}$. The averaged loadunload curve is presented in fig. $4 \mathrm{~b}$. As it can be seen in the figure, this time, the values of the effective indentation modulus did not increase with the static load. The values of $M_{\text {eff }}$ during the initial load-points were about $8.5 \mathrm{GPa}$ and then decreased slightly to $8.2 \mathrm{GPa}$. The unload values of $M_{\text {eff }}$ remained constant within the values of the standard deviation. The mean value of $M_{\text {eff }}$ calculated from all the values obtained during this measurement series was $8.3 \pm 0.4$ GPa and in a very good agreement with that obtained from fitting of eq. 5 to the curve presented in fig. 3b. The results obtained for the indentation modulus of ULK-46 sample are 
about $1 \mathrm{GPa}$ and $2 \mathrm{GPa}$ larger than those obtained for the samples ULK-200 and ULK-350, respectively. These differences are too large to be associated only with the change in the geometries of the tips used.
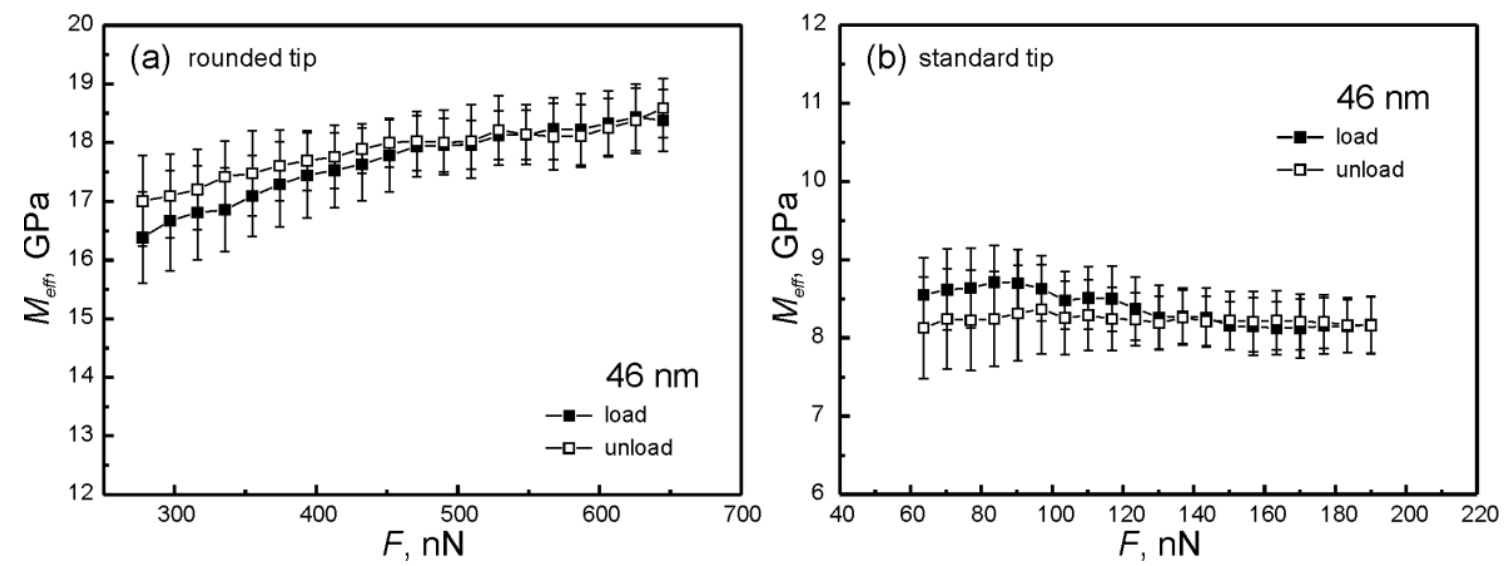

Fig. 4. Values of the effective indentation modulus $M_{\text {eff }}$ determined for the ULK $-46 \mathrm{~nm}$ sample as a function of increasing and decreasing load with (a) rounded and (b) standard sensor tip.

The results presented in figs. 3 and 4 show that the results of the effective indentation modulus obtained for the ULK-46nm sample depend strongly on the measurement conditions. Namely, (i) the values of the indentation modulus obtained with help of the large tips were greater than those determined with tips of radii $\leq 50 \mathrm{~nm}$; (ii) the results obtained with the large rounded tips showed a strong dependence on the applied static load regardless on the load range; (iii) results obtained for $M_{\text {eff }}$ with the tip of radii $\leq 50 \mathrm{~nm}$ showed that tips of small radii yield almost constant values of $M_{\text {eff }}$ when applied at low values of the static loads (iv) increasing the tip-sample interaction volume by using a slightly larger tip and greater values of the static load caused a visible dependence of the effective indentation modulus on the applied load; (v) the results obtained from fitting eq. (7) to curves presented in figs. 3(a), 3(b), and 4(a) yielded results that depended on the tip radius.

Prior to concluding on the development in the pore topology with the increasing thickness of the porous OSG films, we must evaluate the substrate influence on the data obtained for $M_{\text {eff }}$ for all the films tested in this study. Thus we used a dimensionless parameter $\gamma$ that combines the influence of the tip radius $R_{\text {eff }}$, deformation depth $\delta$, and the film thickness $t$ on the effective elastic properties of the probed volume, namely:

$\gamma=\frac{\delta R}{t^{2}}$

To calculate the parameter $\gamma$ we used the corresponding values of the effective tip radius $R_{\text {eff }}$ and film thickness $t$ listed in Tab. II. The deformation of the sample was calculated with help of eq. (4), where the values of the reference contact stiffness were replaced by those determined for thin film samples. The results of our calculations were plotted first as a function of the static load normalized to the maximal value applied during the given measurement series and presented in fig .5(a). Then, we plotted the values of the effective 
indentation modulus determined during each measurement series as a function of the parameter $\gamma$ and showed the resulting curves in fig. 5 (b). Graphs presented in fig. 5 have a mutual legend. We displayed the parameter $\gamma$ on a logarithmic scale for better comparison purposes.
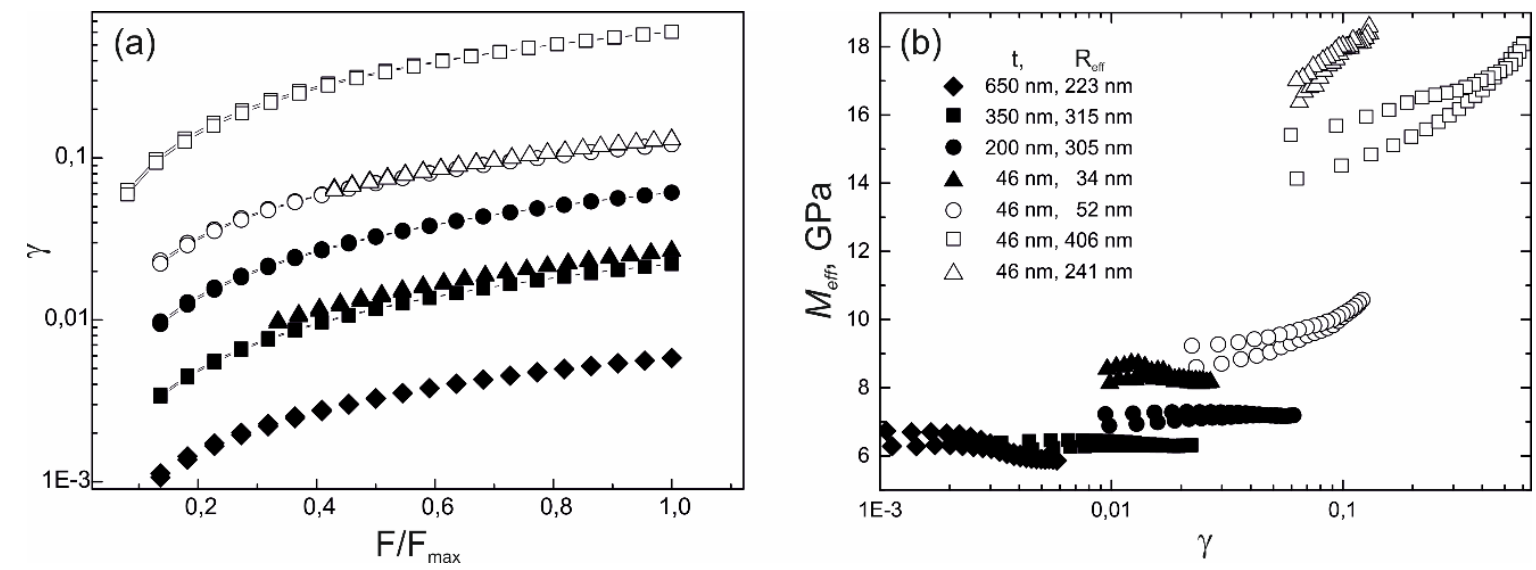

Fig. 5 (a) Values of the parameter $\gamma$ as a function of the normalized static load calculated from results obtained for ULK thin-films with tips of different radii. (b) Plotting the values of the effective indentation modulus as a function of the parameter $\gamma$ helps to visualize the influence of the substrate. $\gamma$ values determined for the ULK film with thickness of $650 \mathrm{~nm}$ were used to determine the level of the substrate influence on the effective elastic properties of the thin film sample.

We used the previously published results obtained for a $650 \mathrm{~nm}$ thin film of porous organosilicate glass to calculate the parameter $\gamma$ to establish the reference for substrate free behavior [42]. The results of those calculations are also shown in fig. 5. According to expectations, the parameter $\gamma$ increases as a function of the applied static load (see fig. 5a). One can see the varying dependence of $\gamma$ on the film thickness and the tip radius. For example, $\gamma$ determined for the $650 \mathrm{~nm}$ film has the lowest values. Then follow the films ULK-350nm and ULK 46nm ( $\mathrm{R}=34 \mathrm{~nm})$. The values of the parameter $\gamma$ determined for these three measurement series are lower than the $\gamma$ values determined for the $46 \mathrm{~nm}$ film with tips $\mathrm{R}=52,241$, and $406 \mathrm{~nm}$. Those three measurement series were most definitely influenced by the elastic properties of the substrate.

The data comparison presented in fig. 5a allowed stating in which case the substrate influence is at its lowest and largest, however, it was still very difficult to state in which case the substrate influence can be neglected. Thus, the data were presented as dependence of the effective indentation modulus as a function of $\gamma$. As it can be seen in fig. $5 \mathrm{~b}$, there is no dependence of the $M_{\text {eff }}$ on parameter $\gamma$ for results obtained for the $650 \mathrm{~nm}$ thin OSG film, as well as for the ULK-350nm, ULK-200nm and ULK-46nm $(\mathrm{R}=34 \mathrm{~nm})$ samples. The results obtained for the ULK-46nm film with the two largest tips are clearly separated. The curve determined for the ULK-46nm $(\mathrm{R}=52 \mathrm{~nm})$ is also influenced by the substrate properties but to a smaller degree than those obtained for ULK-46nm film with the two largest tips.

The results obtained for the ULK -46nm sample show that our simple empirical model for determination of the indentation modulus of thin films can be applied only if the stress fields 
are maintained mainly within the film thickness. To visualize the differences between the stress fields generated under tips of different radii contacting a thin film/substrate system, we have modeled a spherical tip with indentation modulus of $\mathrm{M}_{t}=165 \mathrm{GPa}$ and radius of $\mathrm{R}_{1}=25$ $\mathrm{nm}, \mathrm{R}_{2}=50 \mathrm{~nm}$, and $\mathrm{R}_{3}=100 \mathrm{~nm}$ contacting a $50 \mathrm{~nm}$ thick film with modulus of $\mathrm{M}_{\mathrm{f}}=8 \mathrm{GPa}$ deposited on a elastically isotropic substrate with indentation modulus of $165 \mathrm{GPa}$. The static loads ranged from $50 \mathrm{nN}$ to $1400 \mathrm{nN}$ for the tip radii $\mathrm{R}_{1}$ and $\mathrm{R}_{2}$, and from $200 \mathrm{nN}$ to $3000 \mathrm{nN}$ for tip radius $\mathrm{R}_{3}$. These are standard parameters used during mechanical characterization of materials using AFM or small scale indentation measurements. We used the analytical solution proposed by [56], implemented in software Elastica ${ }^{\circledR}$ by ASMEC to calculate the stress filed distribution in the $z$-direction across the film thickness under the tips of different radii for different values of the applied static load. We calculated the ratio of the stress generated directly under the tip to that at the film-substrate interface. The results are plotted as a function of the applied static load for the three tip radii and presented in fig. 6 . The resulting contact radius to tip radius ratios $a_{c} / R$ are greater those defined as valid for Hertzian based model, which requires that $\frac{a_{c}}{R} \ll 0.05$. In addition, the mismatch between the moduli of the film and the substrate exceeds the model limit. Thus, the values obtained during the calculations for the stress under the indenter are overestimated. Thus, the results predicted by the analytical solution can be treated only as an upper limit. However, they still qualitatively show the behavior of the contact stress-strain filed.

The results of the analytical calculations confirm our expectations. Stress fields created under tips with radii below $50 \mathrm{~nm}$ are contained mostly within the film thickness for the lowest values of the applied loads. The ratio of the stress magnitude at the film surface to that at the film interface $\frac{\sigma_{z, \text { surf }}}{\sigma_{z, \text { interf }}}$ decreases rapidly with the increasing static load. Even a small change in the static load, results in the change of the ratio $\frac{\sigma_{z, \text { surf }}}{\sigma_{z, \text { interf }}}$. For the tips with radii in the range of $100 \mathrm{~nm}$, the resulting contact radius is too large and the resulting stress filed expands beyond the film thickness. As it can be seen, the ratio $\frac{\sigma_{z, \text { surf }}}{\sigma_{z, \text { interf }}}$ varies very little even for relatively large changes in the applied static load.

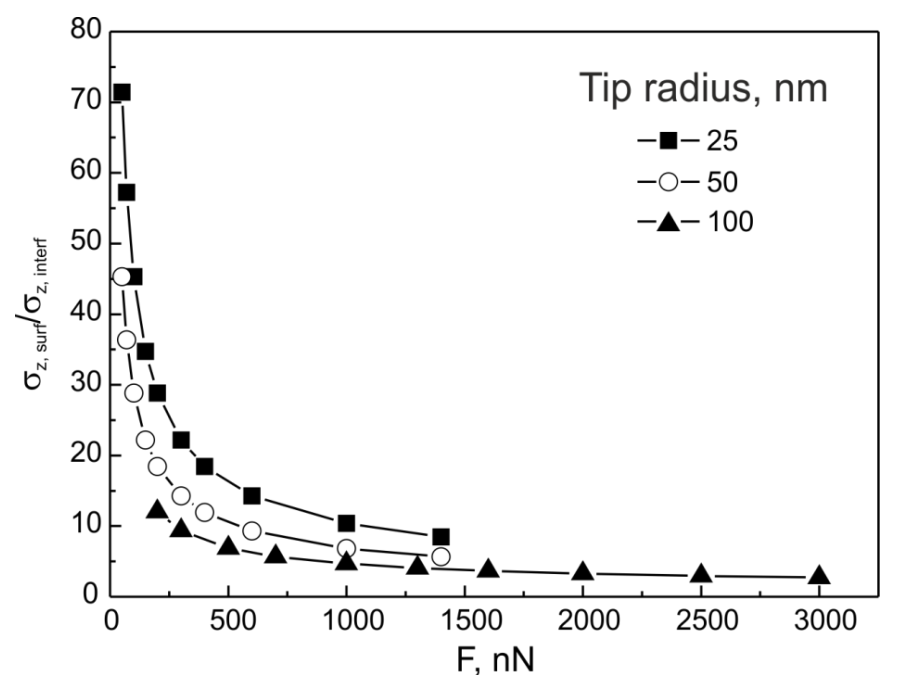


Fig. 6 Ratio of the stress generated directly under the tip at the film surface to that at the filmsubstrate interface calculated for different tip radii as a function of the applied load. Film thickness and indentation modulus were $50 \mathrm{~nm}$ and $8 \mathrm{GPa}$, respectively. The indentation modulus of the substrate was $165 \mathrm{GPa}$.

The results presented in fig. 6 help to understand the discrepancy in the results obtained for the $46 \mathrm{~nm}$ film with help of large tips and those obtained with "standard" AFM tips. The model used for the evaluation of the indentation modulus of thin film can be used only if we can see the "evolution" of the stress field in the film and the substrate. If the relative magnitude of the stress fields at the film surface and its substrate interface remain almost constant the substrate influence is too dominant and our simple empirical model fails to yield correct data. Thus, the data obtained with rounded tips yielded too high values, regardless on the range of the applied load.

\section{Summary and conclusions}

The atomic force acoustic microscopy was used to determine the elastic properties of thin films of porous organosilicate glass. The investigated films had nominally the same porosity of $30 \%$. The thicknesses of the films were $350 \mathrm{~nm}, 200 \mathrm{~nm}$, and $46 \mathrm{~nm}$. Initially, cantilevers with rounded tip sensors were used. The values of the indentation modulus obtained for two thicker films were $6.3 \mathrm{GPa} \pm 0.2 \mathrm{GPa}$ and $7.1 \mathrm{GPa} \pm 0.2 \mathrm{GPa}$ for the $350 \mathrm{~nm}$ and $200 \mathrm{~nm}$ films, respectively.

We have used four tips with different radii to determine the properties of the ULK-46 nm sample. The values obtained for the effective indentation modulus differed depending on the tip radius and on the applied load. To evaluate the influence of the substrate on the effective indentation modulus of the thin-film system, we introduced a parameter $\gamma$ combining the ratios of the tip radius and the deformation depth to the film thickness. The comparison of the $\gamma$ values determined for different samples revealed that we can use this parameter to separate the results into free of the substrate influence, dominated by the substrate properties, and influenced by the substrate elastic properties. The separation is especially visible when the values of the effective indentation modulus $M_{\text {eff }}$ are plotted as a function of the parameter $\gamma$.

The AFAM data obtained for the ULK $-46 \mathrm{~nm}$ sample with tips of radii $\leq 55 \mathrm{~nm}$ yielded in two independent measurements series values of the indentation modulus of about $8.3 \mathrm{GPa}$. In the first measurement set the applied forces ranged from $200 \mathrm{nN}$ to $1400 \mathrm{nN}$ and the values of the effective indentation modulus increased as a function of the applied load, indicating the substrate influence. As we knew the film thickness and the elastic properties of the substrate, we used an empirical model to calculate the elastic properties of the film. The result of 8.3 $\mathrm{GPa} \pm 0.1 \mathrm{GPa}$ was in very good agreement with those obtained in the second series of the AFAM measurements with use of a "standard" tip. In this case, the applied static loads were lower than $200 \mathrm{nN}$ and the obtained results for $M_{\text {eff }}$ did not show substrate influence. The mean value calculated for this measurement series was $8.3 \mathrm{GPa} \pm 0.4 \mathrm{GPa}$.

The substrate free results obtained for the ulk-46nm sample followed the trend observed for the ULK -200nm and ULK -350 nm samples. The effective indentation modulus of the tested 
films increased with their decreasing thickness. Such change of the elastic properties suggests a change in the pore topology, such as evolution of the pore size and structure with film thickness. The results obtained in this study combined with those published previously [42] indicate that studies of mechanical properties of porous materials with help of AFM-based methods can provide indirect but valuable information on the relative changes in the pore topology.

The results obtained for the ULK $-46 \mathrm{~nm}$ film with help of rounded and "standard" AFM tips allowed learning more about the interaction scale in nano-scale contact mechanics. For example, to characterize mechanical properties of nano-thin films, not only the range of the applied forces and the size of the indenter, but also the evolution of the stress filed in the film as a function of the applied loads have to be taken into account.

\section{Acknowledgments}

This work was part of the project KO-3825/1-1 "Nanoscale properties of active polymer/organic-based electronic components by atomic force acoustic microscopy" supported by DFG (Deutsche Forschungsgemeinschaft). In addition, S. M. and T. S. would like to thank the Center for Integrated Nanotechnologies, an Office of Science User Facility operated for the U.S. Department of Energy (DOE) Office of Science by Los Alamos National Laboratory (Contract DE-AC52-06NA25396) and Sandia National Laboratories (Contract DE-AC04-94AL85000).

\section{Literature}

1. A. A. Volinsky, J. B. Vella, W. W. Gerberich, Thin film solids 429 (2003) 201-210

2. C. M. Flannery, T. Wittkowski, K. Jung, B. Hillebrands, and M.R. Baklanov, Applied Physics Letters 80 (2002) 4594-4596

3. L.L. Chapelon, J. Vitiello, D. Neira, J. Torres, J.C. Royer, D. Barbier, F. Naudin, G. Tas, P. Mukunhan, J. Clerico , Microelectronic Engineering 83 (2006) 2346-2350

4. K.P. Mogilnikov and M.R. Baklanov, Electrochemical and solid-state letters 5, (2002) F-29-F31

5. W.C. Oliver and G. M. Pharr, Journal of Material Research 7 (1992) 1564

6. G. Binning and C. F. Quate, Physical Review Letters 56 (1986) 930

7. T. Chudoba, N. Schwarzer, F. Richter, Surface and Coatings Technology 154 (2002) 140-151

8. K. B. Yeap, M. Kopycinska-Müller, U. Hangen, T. Wyrobek, R. Hübner, S. Niese, and E. Zschech, Philosophical Magazine, available on-line DOI:10.1080/14786435.2012.669071, 2012

9. M. F. Doerner and W. D. Nix, Journal of Material Research 1 (1986) 601-609

10. R. B. King, International Journal of Solids Structures 23 (1987) 1657-1664

11. H. Gao, Ch.-H. Chiu, and J. Lee, International Journal of Solids Structures 29 (1992) 2471-2492

12. M.V. Swain and J. Mencik, Thin Solid Films (1994) 204-211

13. N. E. Waters, Brit. Journal of Applied Physics 16 (1965) 557 - 563 
14. R. Saha and W. D. Nix, Acta Materialia 50 (2002) 23-38

15. N. Schwarzer, M. Whittling, M. Swain, and F. Richter, Thin Solid Films 270 (1995) $371-375$

16. N. Schwarzer, T. Chudoba, D. Billep, and F. Richter, Surface and Coatings Technology 116-119 (1999) 244-252

17. T. Chudoba, N. Schwarzer, and F. Richter, Surface and Coatings Technology 127 (2000) 9-17

18. H. Li and J. J. Vlassak, Journal of Material Research 24 (2009) $1114-1126$

19. S. J. Bull, Philosophical Magazine, DOI: 10.1080/14786435.2014.909612

20. O. Sahin, S. Maganov, C. Su, C. F. Quate, and O. Solgaard, Nature Nanotechnology 2 (2007) 507

21. T. J. Young, M. A. Monclus, T. L. Burnett, W. R. Broughton, S. L. Ogin, and P. A. Smith, Measuremnts Science Technology 22 (2011) 125703

22. M. E. Dokukin and I. Sokolov, Langmuir 28 (2012) 160160

23. P.-E. Mazeran and J.-L. Loubet, Tribology Letters 7 (1999) 199-212

24. D. G. Yablon, J. Grabowski, and I. Chakraborty, Measurement Science and Technology 25 (2014) 055402

25. A. B. Kos, J. P. Killgore, and D. C. Hurley, Measurement Science and Technology 25 (2014) 025405,

26. B. J. Rodriguez, C. Callahan, S. V. Kalinin, and R. Proksch, Nanotechnology 18 (2007) 475504

27. S. Jesse, S. V. Kalinin, R. Proksch, A. P. Baddorf, and B. J. Rodriguez, Nanotechnology 18 (2007) 435503

28. A. L. Weisenhorn, P. Maivald, H.-J. Butt, P. K. Hansma, Physical Review B 45 (1992) 11226

29. A. Rosa-Zeiser, E. Weilandt, S. Hild, and O. Marti, Measurements Science and Technology 8 (1997) 1333

30. R.E. Geer, O.V. Kolosov, G. A. D. Briggs, and G. S. Shekhawat, Journal of Applied Physics 81 (2002) 4549

31. K. Yamanaka, Y. Maruyama, T. Tsuji, and K. Nakamoto, Appllied Physics Letters 78 (2001) 1939

32. U. Rabe, K. Janser, and W. Arnold, Review of Scientific Instruments 67 (1996) 3281

33. D. C. Hurley, "Measuring mechanical properties on the nanoscale with contact resonance force microscopy methods" in "Scanning Probe Microscopy of Functional Materials: Nanoscale Imaging and Spectroscopy", Eds. S. V. Kalinin and A. Gruverman, Springer Science+Bussiness Media, LLC 2010, DOI 10.1007/978-14419-7167-8_4

34. G. Stan and R. F. Cook, Nanotechnology 19 (2008) 235701

35. D.C. Hurley, K. Shen, N. M. Jennett, J. A. Turner, Journal of Applied Physics 94 (2003) 2347

36. D. C. Hurley, M. Kopycinska-Müller, A. B. Kos, R. H. Geiss, Measurement Science and Technology 16 (2005) 2167

37. M. Kopycinska-Müller, R. H. Geiss, J. Müller, and D.C. Hurley, Nanotechnology 16 (2005) 703 
38. D. C. Hurley, M. Kopycinska-Müller, E. D. Langois, A. B. Kos, N. Barbossa, Applied Physics Letters 89 (2006) 021911

39. M. Muraoka and S. Komatsu, Nanoscale Research Letters 6:33 (2011)

40. M. Kopycinska-Müller, A. Striegler, A. Hürrich, B. Köhler, N. Meyendorf, and K.-J. Wolter, Mater. Res. Soc. Symp. Proc. 1185, in Probing Mechanics at Nanoscale Dimensions, edited by N. Tamura, A. Minor, C. Murray, L. Friedman, 1185-II09-04, 2009

41. G. Stan, S. W. King, and R. F. Cook, Journal of Material Research 24 (2009) 29602964

42. M. Kopycinska-Müller, K. B. Yeap, S. Mahajan, B. Köhler, N. Kuzeyeva, T. Müller, E. Zschech, K.-J. Wolter, Nanotechnology, 24, (2013) 355703

43. G. Stan, R. S. Gates, P. Kavuri, J. Torres, D. Michalak, C. Edge, J. Bielefeld, and S. W. King, Applied Physics Letters 105 (2014) 152906

44. D. Zhao, J. Feng, Q. Huo, N. Melosh, G. F. Fredrickson, B.F. Chmelka, G.D. Stucky, Science 279 (1998) $548-552$

45. U. Rabe, S. Amelio, E. Kester, V. Scherer, S. Hirsekorn, and W. Arnold, Ultrasonics 38 (2000) 430

46. U. Rabe, M. Kopycinska-Müller, S. Hirsekorn, "AFAM technique and calibration" in: Acoustic Scanning Probe Microscopy, F. Marinello, D. Passeri, E. Savio (Eds.) Springer-Verlag, Series on Nanoscience and Technololgy, 2012

47. J. J. Vlassak and W. D. Nix, Philosophical Magazine A 67 (1993) 1045

48. J.J. Vlassak, M. Ciavarella, J. R. Barber, X. Wang, Journal of Mechanics and Physics of Solids 51 (2003)1701-1721

49. M. Kopycinska-Müller, A. Striegler, R. Schlegel, N. Kuzeyeva, B. Köhler, and K.-J. Wolter, Rev. Sci. Instrum. 83, 043703 (2012)

50. U. Rabe, S. Amelio, M. Kopycinska, S. Hirsekorn, M. Kempf, M. Goecken, and W. Arnold, Surface and Interface Analysis 33 (2002) 65

51. J. E. Sader, I. Larson, P. Mulvaney, and L. R. White, Review of Scientific Instruments 66 (1995) 3789

52. M. Kopycinska-Mueller, R.H. Geiss, D. C. Hurley, Ultramicroscopy 106 (2006) 466474

53. D. Passeri, A. Bettucci, M. Germano, M. Rossi, A. Alippi, S. Orlanducci, M. L. Terranova, and M. Ciavarella, Review of Scientific Instruments 76 (2005) 093904

54. M. Kopycinska-Müller, On the elastic properties of nanocrystalline materials and the determination of elastic properties on a nanoscale using the atomic force acoustic microscopy technique, $\mathrm{PhD}$ thesis, Science and Technical Faculty III, Saarland University and IZFP report No. 050116-TW (2005)

55. B. V. Derjaguin, V. M. Müller, and Yu P. Toporov, Journal of Colloid and Interface Science 53 (1975) 314

56. N. Schwarzer, F. Richter, and G. Hecht, Surface and Coatings Technology 114 (1999) 292-304 